\title{
ASPECTOS CONCEITUAIS DE TERMINOLOGIAS RELATIVAS ÀS ABORDAGENS COGNITIVAS E COMPORTAMENTAIS
}

\section{CONCEPTUAL ASPECTS OF TERMINOLOGIES CONCERNING COGNITIVE AND BEHAVIORAL APPROACHES}

'Wildson Cardoso Assunção.

\section{RESUMO}

Introdução: Historicamente a Psicologia esteve relacionada à filosofia, ao longo dos anos a psicologia foi ganhando mais autonomia no que diz respeito às formações e aos profissionais que nela atuavam. A terminologia TCC é bastante usada em artigos científicos e livros, sendo mais encontrada do que PCC, podendo gerar confusão sobre seus conceitos e aplicabilidades, dessa forma, se faz necessário conceituar e definir essas terminologias de forma mais clara. Objetivos: Definir aspectos conceituais de terminologias relativas às abordagens cognitivas e comportamentais, com base na reflexão de suas teorias e outros estudos. Metodologia: Para construção dessa pesquisa foi escolhida a revisão narrativa da literatura. Esse método envolve uma ampla gama de recuperação de publicações apropriadas para descrever e discutir determinados conceitos sob o ponto de vista teórico ou contextual. Resultados e discussão: Logo após um breve levantamento da literatura houve a leitura dos resumos, buscando responder à seguinte questão: "Qual seria a definição mais precisa em relação às abordagens cognitivas e comportamentais?". Foi identificada uma variedade de teorias e suas aplicabilidades, considerando eixos de pesquisas dentro das seguintes áreas: Ciências Cognitivas, Psicologia, Teorias, Terapias. Conclusão: A presente revisão narrativa da literatura conseguiu definir algumas das terminologias levantadas através de seus contextos históricos, no entanto, talvez não seja possível encontrar definições mais claras, devido às abordagens serem distintas e ao mesmo tempo muito parecidas em alguns aspectos, por outro lado, entende-se que através de novas pesquisas seja possível estabelecer quais são as divergências, ou quais são suas aplicabilidades.

Descritores: Psicologia. Terapia CognitivoComportamental. Teorias Cognitivas.

\section{ABSTRACT}

Introduction: Historically, psychology has been related to philosophy, over the years psychology has been gaining more autonomy with respect to the formations and the professionals who worked in it. CBT terminology is widely used in scientific articles and books, being more found than PCC, which can lead to confusion about its concepts and applicability, so it is necessary to conceptualize and define these terminologies more clearly. Objectives: Define conceptual aspects of terminologies related to cognitive and behavioral approaches, based on the reflection of their theories and other studies. Methodology: For the construction of this research was chosen the narrative review of the literature. This method involves a wide range of retrieval of appropriate publications to describe and discuss certain concepts from a theoretical or contextual point of view. Results and discussion: After a brief survey of the literature, the abstracts were read, seeking to answer the following question: "What would be the most precise definition in relation to cognitive and behavioral approaches?". A variety of theories and their applicability were identified, considering research axes within the following areas: Cognitive Sciences, Psychology, Theories, Therapies. Conclusion: The present narrative review of the literature has managed to define some of the terminologies raised through its historical contexts, however, it may not be possible to find clearer definitions, because the approaches are distinct and at the same time very similar in some aspects, on the other hand, it is understood that through further research it is possible to establish what the divergences are or what their applicability is.

Descriptors: Psychology. Cognitive-behavioral Therapy. Cognitive Theory.
${ }^{1}$ Psicólogo pela Universidade de Gurupi UnirG. Pós-Graduado em Psicologia Organizaciona e do Trabalho pela Faculdade de Ciências Sociais Aplicadas de Marabá - FACIMAB

Pós-Graduando

Terapia CognitivoFACItamental FACIMAB.

E-mail:

wildson.se@outlook.com

ENDEREÇO PARA CORRESPONDÊNCIA:

Wildson

Assunção. Cardoso Secretaria de Assistência Social / Centro de Referência

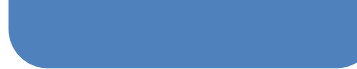

\section{INTRODUCAO}

Historicamente a Psicologia esteve ligada à filosofia, foi oficialmente reconhecida como uma ciência autônoma em 1879, no laboratório de Psicologia Experimental fundado por Wilhelm Wundt, na cidade Alemã de Leipzig ${ }^{1}$.
De acordo com o autor, essa nova psicologia espalhou-se pela Europa e depois para a América, especialmente nos Estados Unidos, onde mais tarde haveria novas correntes de pensamento que embasariam ainda mais a psicologia como uma ciência. 
Recebido: 05 agosto 2018. Aceito: 17 dezembro 2018. Revista Amazônia Science \& Health - 2018 Out/Dez.
Assunção, WC

ASPECTOS CONCEITUAIS DE TERMINOLOGIAS RELATIVAS ÀS ABORDAGENS COGNITIVAS E COMPORTAMENTAIS
No Brasil apesar de haver registros históricos da atuação da psicologia, ela só fora regulamentada em 1962, através da lei no 4.119, em 27 de Agosto daquele ano. Essa lei dispõe sobre os cursos de formação em psicologia e regulamenta a profissão de psicólogo.

Antes desse marco histórico, a psicologia era mais aprofundada como uma especialização de Filosofia e era exercida principalmente por médicos e profissionais relacionados à educação ${ }^{2}$.

Logo após a regulamentação da Psicologia, através da lei n 5.766/71 o Conselho Federal de Psicologia - CFP foi criado, junto dos conselhos regionais. Ao longo dos anos a psicologia foi ganhando mais autonomia no que diz respeito às formações e aos profissionais que nela atuavam, bem como suas subáreas de atuação.

As áreas de atuação da psicologia são relativas às abordagens $e$ modelos teóricos $e$ metodológicos que podem ser escolhidos como ferramentas de atuação a depender de cada psicólogo.

Dentre as abordagens da psicologia existem, por exemplo, o behaviorismo (clássico), desenvolvido por e John B. Watson, que tinha como foco os comportamentos observáveis, posteriormente tornando-se o behaviorismo radical, aprimorado por Burrhus Frederic Skinner ${ }^{3}$.

Após uma série de inovações e revoluções no campo da psicologia surge a Psicologia Cognitivo-Comportamental - PCC, devido uma necessidade de rompimento da psicologia tradicional da época ${ }^{4}$.

A PCC surgira nos anos 50 e apesar de ainda ser uma recente área, sua influência cresceu rapidamente. De acordo com os autores a PCC foi criada através das pesquisas realizadas por Aaron Temkin Beck, que foi o pioneiro nessa nova abordagem, tendo influência principalmente da psicanálise de alguns pensadores pós-Freudianos ${ }^{5}$.

A psicanálise, por sua vez, tinha como objeto de estudo $o$ inconsciente $e$ as estruturas que influenciavam na personalidade 6 . No entanto, a PCC colocava como objeto de estudo principalmente a cognição, o comportamento e suas varáveis.

Ao longo do tempo surgiram outras teorias relacionadas aos modelos de intervenção da PCC. $\mathrm{Na}$ atualidade a literatura apresenta diferentes nomenclaturas ao tratar das abordagens cognitivas e comportamentais, como a Terapia CognitivoComportamental, que seria um modelo teóricoprático da PCC, baseado em evidências, sendo usado principalmente em tratamento de transtornos mentais ${ }^{7}$.

A terminologia TCC existe devido à junção de saberes das teorias cognitivas e teorias comportamentais, dessa forma a separação entre "terapia cognitiva" e "terapia comportamental" existe apenas para fins didáticos ${ }^{8}$.
Abordagens como o estruturalismo e o funcionalismo deram origem a estudos relativos à psicologia cognitiva, que trata das questões relacionadas a cognições, incluindo relações entre percepção, aprendizagem, memória e pensamentos

- Entende-se por terminologia um conjunto de termos específicos de uma determinada especialidade, sendo que cada área do conhecimento faz seu uso de formas diferentes ${ }^{10}$. Em outras palavras, no contexto científico, as terminologias são um tipo de código linguístico que definem e dão nomes a determinadas áreas.

A nomenclatura TCC é bastante usada em artigos científicos e livros, sendo mais encontrada, dessa forma, imagina-se que ainda podem existir divergências entre seus conceitos e aplicabilidades. Através do exposto, o objetivo principal desse artigo foi definir aspectos conceituais de terminologias relativas às abordagens cognitivas e comportamentais, com base na reflexão de suas teorias e outros estudos.

\section{MATERIAL E METODOS}

Para construção dessa pesquisa foi escolhida a revisão narrativa da literatura. Esse método envolve uma ampla gama de recuperação de publicações apropriadas para descrever e discutir determinados conceitos sob o ponto de vista teórico ou contextual ${ }^{11}$.

De acordo com os autores esse método permite uma análise da literatura considerando a interpretação e análise critica do autor. Esse método possibilita também ser mais breve e focado em uma resolução breve de algum questionamento, não precisando, portanto, utilizar de esgotamento das buscas nas bases de dados ${ }^{12}$.

Não foi delimitado um período específico para as buscas, devido à pesquisa buscar conceitos atuais e outrora explorados em busca de definições que possibilitassem reflexões mais precisas. Em relação aos idiomas, foram utilizados o Português e Inglês e Espanhol.

As bases de dados utilizadas foram a Biblioteca Virtual em Saúde (BVS), incluindo seus portais indexados, Google Acadêmico, artigos de periódicos em formato de jornal eletrônico, dissertações, teses de mestrado e doutorado, trabalhos de conclusão de curso, livros e outras informações que trouxessem conteúdos pertinentes à pesquisa.

Os títulos e os resumos de todos os artigos foram lidos primeiros antes de uma seleção mais criteriosa. Com base nesta ação, foi criada uma lista de pesquisas que possivelmente seriam incluídos nesse estudo. Após isso foram aplicados os critérios de inclusão e exclusão, posteriormente a construção da presente pesquisa. 
Recebido: 05 agosto 2018. Aceito: 17 dezembro 2018. Revista Amazônia Science \& Health - 2018 Out/Dez.
Assunção, WC

ASPECTOS CONCEITUAIS DE TERMINOLOGIAS RELATIVAS ÀS ABORDAGENS COGNITIVAS E COMPORTAMENTAIS
A escolha das terminologias foi pensada com base nos termos encontrados no levantamento inicial da bibliografia, considerando suas divergências. Foram utilizados os seguintes descritores, traduzidos conforme a seleção das pesquisas, em Português, Inglês e Espanhol: (Psicologia Cognitiva ou Psicologia Comportamental; Terapia Cognitiva ou Terapia Comportamental; Teorias cognitivas ou Teorias comportamentais), e assim sucessivamente.

Os critérios de inclusão adotados para seleção de artigos precisariam ser publicados em periódicos científicos (para o caso de artigos fora das bases de dados citadas acima); precisariam publicados por órgãos oficiais, na língua Portuguesa ou Inglesa ou Espanhola; Precisaram ter em seu conteúdo alguma forma de definição da PCC e suas abordagens; e precisariam considerar aspectos históricos, de maneira que fosse possível considerar definições a partir disso.

Foram excluídos artigos incompletos, que não estavam hospedados em sites oficiais; e que não estavam, de alguma forma, relacionados às palavras chaves ou semelhantes.

\section{RESUTADOS E DISCUSSAOO}

Após um breve levantamento bibliográfico foram selecionados, na íntegra, os possíveis artigos, pesquisas e livros pertinentes às temáticas aqui levantadas. A partir dessa avaliação, foi realizada a construção metodológica da pesquisa.

No breve levantamento bibliográfico no portal da BVS, aplicados os critérios, foram encontrados 13 pesquisas que continham a definição de "psicologia cognitivo-comportamental" e 23 pesquisas com a terminologia "terapia cognitivocomportamental, no entanto, apenas 7 desses artigos foram incluídos por causa de seus expressos conteúdos; foram encontradas 89 pesquisas com a terminologia "terapias cognitivas" e 137 contendo "terapias comportamentais".

Logo após esse breve levantamento houve a leitura dos resumos, buscando responder à seguinte questão: Qual seria a definição mais precisa em relação às abordagens cognitivas e comportamentais?.

A pergunta foi respondida, considerando as definições conceituadas na literatura, que apesar de variadas, seguiam uma mesma linha, que possibilitava espaço para definições mais claras.

Foi identificada uma variedade de teorias e suas aplicabilidades, considerando eixos de pesquisas dentro das seguintes áreas: Ciências Cognitivas, Psicologia, Teorias, Terapias.

No decorrer da presente pesquisa, não foram encontrados estudos que discutisse aspectos conceituais relativos às terminologias, no entanto, é interessante notar que praticamente todas as pesquisas recuperadas abordaram conceitos históricos, que podem, impreterivelmente, serem usados como base para definir e conceituar as terminologias.

Este fato demonstra que apesar das pesquisas serem escassas, pode haver reflexão em cima das teorias e de seus conceitos originais.

As pesquisas mostram evidências na eficácia das aplicações das terapias e dos postulados científicos das "ciências cognitivas", que, por se tratar de "um conjunto de disciplinas, mas por possuírem objetivos muito parecidos ${ }^{13}$.

Considerando a divergência dos conceitos, bem como sua discrepância no tocante à forma e ao período das discussões e definições, o método escolhido de revisão narrativa pode possibilitar descrições e discussões acerca do "estado da arte", ou seja, discussões do mais alto desenvolvimento de uma técnica ou área científica.

As hipóteses iniciais eram que não haviam definições precisas de terminologias relativas às abordagens cognitivas e comportamentais.

Essas definições parecem se confundir devidas suas nomenclaturas ou devido sua etimologia, por possuírem caracterizações muito amplas, não havendo uma linha divisória clara entre as várias "gerações" de psicoterapias baseadas em evidências e TCC.

As ciências cognitivas são um "conjunto de disciplinas", que incluem a psicologia cognitiva, devido a isso, existe até hoje dificuldades em estabelecer definições claras em relação as terminologias de cada área ${ }^{14}$.

Em relação a essa discussão, havia dificuldade para encontrar essas definições de forma consistente, devido à semelhança entre a amplitude das abordagens ${ }^{15}$.

Dessa forma, conforme os estudos desses autores não existem definições padronizadas em relação às terminologias, no entanto, os estudos desenvolvidos buscam conceituar algumas terminologias, como quando mostram que Terapia Comportamental aparece na literatura também como "Terapia de Modificação do Comportamento" e também como Terapia CognitivoComportamental.

Cada autor parece usar uma definição, como quando usam o termo TCC para se referir a tudo que foi desenvolvido nessa área, enquanto outros utilizam apenas para descrever as intervenções ${ }^{15}$.

As divergências apresentadas na literatura variaram ocorreram no sentido dos objetivos dos estudos pesquisados.

Quanto às terminologias, foi encontrado conteúdo subjetivo de definição, a depender do foco das pesquisas ou de seus segmentos teóricos.

Consideramos essas divergências aceitáveis do ponto de vista cientifico, por compreendermos que tudo o que foi discutido aqui ocorreu em 
épocas diferentes, em lugares diferentes e em contextos diferentes.

A tabela a seguir demonstra de forma mais clara o conceito geral das terminologias desse presente estudo, com base na reflexão de suas teorias.

Tabela 1: Definição das terminologias

TERMINOLOGIA

Ciências cognitivas

Ciências comportamentais

Psicologia cognitiva

Psicologia comportamental

PCC

TCC

Teorias cognitivas

Terapias cognitivas

Terapias comportamentais

\section{DEFINIÇÃO}

Conjunto de disciplinas como a filosofia, psicologia, linguística e etc. que seguem um mesmo principio e que em geral envolve a cognição.

Conjunto de disciplinas que estudam os diversos conceitos de comportamento humano, sendo composta especialmente pela psicologia, sociologia, antropologia, etc.

Estudo das cognições, que também é uma disciplina das Ciências Cognitivas.

Ciência que estuda o comportamento humano e que possui diversas abordagens que seguem um mesmo princípio.

Especialidade da psicologia, baseada na aplicação de princípios básicos e teóricos.

Aplicação prática da Psicologia CognitivoComportamental.

Conceito pluridisciplinar que envolve teorias desde a Psicologia Cognitiva, Antropologia Cognitiva, Sociologia Cognitiva e Biologia. Base das Ciências Cognitivas.

Conjunto de intervenções que se baseiam na cognição (Focada em compreender processos mentais idiossincráticos e disfuncionais).

Método de aplicação clinica de ciências comportamentais, especialmente da psicologia.
A tabela 2 possibilita a compreensão, em um resumo do que foi discutido, com base nos pressupostos teóricos de atuação de cada área, aqui é possível perceber como cada uma dessas áreas citadas na tabela 1 atua. 
Tabela 2: Definição das áreas e seus objetivos de atuação.

\begin{tabular}{|c|c|}
\hline ÁREA & OBJETIVOS \\
\hline Ciências Cognitivas & $\begin{array}{l}\text { Utiliza como base as teorias cognitivas em seu conjunto } \\
\text { de disciplinas para produzir conhecimentos científicos, } \\
\text { relacionados à cognição. }\end{array}$ \\
\hline Ciências Comportamentais & $\begin{array}{l}\text { Utiliza como base as teorias comportamentais em seu } \\
\text { conjunto de disciplinas, especialmente a psicologia. }\end{array}$ \\
\hline Psicologia Cognitiva & $\begin{array}{l}\text { Especialidade da psicologia, que formula base teórica } \\
\text { para a prática. }\end{array}$ \\
\hline Psicologia Comportamental & $\begin{array}{l}\text { Especialidade da psicologia, que formula base teórica } \\
\text { para a prática. }\end{array}$ \\
\hline PCC & $\begin{array}{l}\text { Especialidade da psicologia, que formula base teórica } \\
\text { para a prática. }\end{array}$ \\
\hline TCC & $\begin{array}{l}\text { Aplicação prática da Psicologia Cognitivo- } \\
\text { Comportamental. }\end{array}$ \\
\hline Teorias Cognitivas & $\begin{array}{l}\text { Utiliza os saberes de diversas áreas para formular teorias } \\
\text { que possibilitem desenvolvimento científico }\end{array}$ \\
\hline Terapias Cognitivas & $\begin{array}{l}\text { Aplicação prática da Psicologia Cognitiva e outras } \\
\text { terapias, como a TCC e suas variáveis. }\end{array}$ \\
\hline Terapias Comportamentais & Aplicação prática da Psicologia Comportamental. \\
\hline
\end{tabular}

A figura 1 abaixo representa uma sintetização das tabelas 1 e 2 , de forma cronológica-conceitual dessas abordagens.

Figura 1: Evolução cronológica-conceitual das abordagens cognitivas e comportamentais

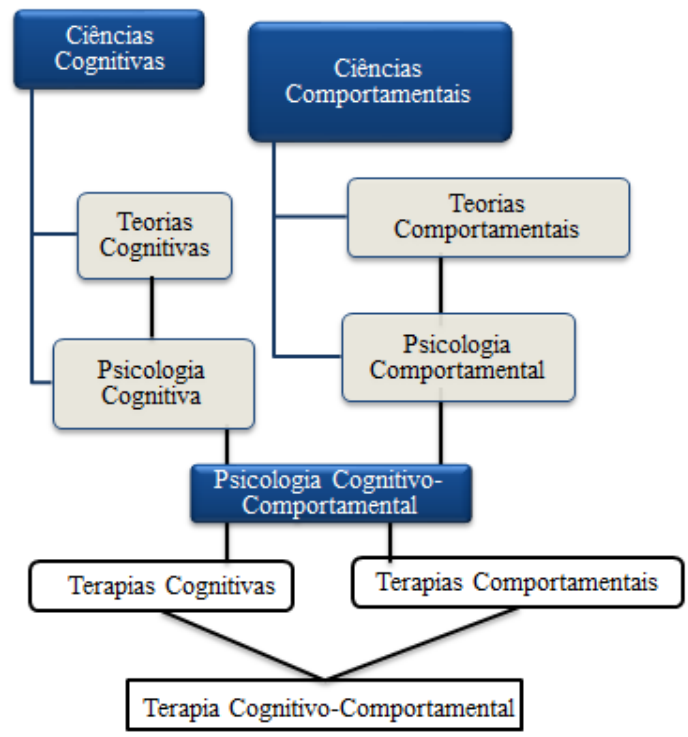

\section{CONCLUSAOO}

O artigo conseguiu alcançar os objetivos de definir algumas das terminologias das abordagens com base na reflexão de suas teorias e de pesquisas científicas que exploraram esses conceitos.

Considera-se que a definição de uma terminologia ou nomenclatura é um pressuposto básico para 0 início de qualquer pesquisa científica, levando em conta de que cada pesquisa precisa se situar relação a uma teoria ou posicionamento adotado para produzir conteúdos científicos.

Esta revisão narrativa da literatura foi necessária por dispor de um método de buscas mais diversificado das reflexões, com o intuito de se aproximar mais das reais definições das áreas levantadas.

De qualquer forma houve uma limitação, por se tratar de um pequeno recorte na literatura, havia uma falta de pesquisas com essa perspectiva e poucas pesquisas que traziam definições objetivas, sendo em sua grande maioria eram pesquisas realizadas na língua 
Inglesa e Espanhola, isso se deve também aos conceitos que foram construídos ao longo do tempo pelas próprias abordagens.

Apesar de não existirem essas definições padronizadas e oficializadas para diversas terminologias relativas às abordagens cognitivas e comportamentais, é possível, através de um contexto histórico, resgatar algumas das ideias presentes nas iniciativas dos movimentos que deram origem a esses ramos científicos.

São necessários mais estudos que investiguem a fundo essas e outras abordagens cognitivas e comportamentais na tentativa de estabelecer uma definição mais aproximada do que a área de estudo realmente objetiva.

Talvez não seja possível encontrar definições mais claras, como visto no levantamento bibliográfico, trata-se de abordagens distintas, mas muito parecidas em suas teorias, métodos e aplicações.

Por outro lado, compreendemos que através de novas pesquisas seja possível estabelecer quais são as divergências, como se define sua teoria, ou quais são suas aplicabilidades, de forma que possa haver clareza para os futuros pesquisadores e comunidade científica.

Essa investigação é tida como importante devido suas possibilidades de engajamento, visto que quando uma abordagem é mais clara, pode haver maiores interesses de pesquisa na área, bem como promoção de seu desenvolvimento.

\section{REFERËNCIAS}

1 Soares, Antonio Rodrigues. (2010). A Psicologia no Brasil. Psicologia: Ciência e Profissão, 30(spe), 8-41.

2 Chaves, Antônio Marcos. (1992). 30 anos de regulamentação. Psicologia: Ciência e Profissão, 12(2), 4-9.

3 Zilio, D. (2010). A Natureza Comportamental da Mente: Behaviorismo radical e filosofia da mente. São Paulo: Cultura.

4 Barbosa, J. I. C. \& Borba, A. (2010). O surgimento das terapias cognitivocomportamentais e suas consequências para 0 desenvolvimento de uma abordagem clínica analítico-comportamental dos eventos privados. Revista Brasileira de Terapia Comportamental e Cognitiva, 12 , 60- 79.

5 Wright, J. H., Basco, M. R., \& Thase, M. E. (2008). Princípios básicos da terapia cognitivocomportamental. Porto Alegre, RS: Artmed.
6 Rosa, M. D. \& Domingues, E. (2010). O método na pesquisa psicanalítica de fenômenos sociais e políticos: a utilização da entrevista e da observação. Psicologia \& Sociedade, 22(1), 180188.

7 Beck, J. S. (2013). Terapia CognitivaComportamental: teoria e prática. $2^{\mathrm{a}}$ Ed. Porto Alegre. Artmed. $413 p$.

8 Knapp, Paulo, \& Beck, Aaron T. (2008). Fundamentos, modelos conceituais, aplicações e pesquisa da terapia cognitiva. Brazilian Journal of Psychiatry, 30(Suppl. 2).

9 Sternberg, R. J. (2016). Cognitive Psychology. $3^{a}$ Ed. Michigan. Wadsworth, Cengage Learning. $680 \mathrm{p}$.

Neufeld, Carmem Beatriz, Brust, Priscila Goergen, \& Stein, Lilian Milnitsky. (2011). Bases epistemológicas da psicologia cognitiva experimental. Psicologia: Teoria e Pesquisa, 27(1), 103-112.

10 Dias, C. A. (2000) Terminologia: conceitos e aplicações. Ci. Inf., Brasília, v. 29, n. 1, p. 90-92.

11 Sallum, Ana Maria Calil, Garcia, Dayse Maioli, \& Sanches, Mariana. (2012). Dor aguda e crônica: revisão narrativa da literatura. Acta Paulista de Enfermagem, 25(spe1), 150-154.

12 Rother, Edna Terezinha. (2007). Revisão sistemática $X$ revisão narrativa. Acta Paulista de Enfermagem, 20(2)..

13 Rangé, B. P; Falcone, E. M. O. \& Sardinha, A. (2007). História e panorama atual das terapias cognitivas no Brasil. Revista Brasileira de Terapias Cognitivas, 3(2).

14 Gauer, G., et al. (2018). Psicologia Cognitiva: Teoria, Modelos e Aplicações. $1^{\underline{a}}$ Ed. SINOPSYS. 256.

15 Hayes, S. C. \& Pistorello, J. (2015) Terapias comportamentais de terceira geração: guia para profissionais / organizado por Paola LucenaSantos, José Pinto-Gouveia e Margareth da Silva Oliveira... [et al.] - Novo Hamburgo: Sinopsys. 